\title{
OBSERVATIONS OF ORDERED ION BEAMS IN CRYRING
}

\author{
H. Danared, A. Källberg, K.-G. Rensfelt and A. Simonsson \\ Manne Siegbahn Laboratory, Frescativägen 24, S-104 05 Stockholm, Sweden
}

\begin{abstract}
A sudden drop in momentum spread of electron-cooled beams of highly charged $\mathrm{Ni}$ and Xe ions, similar to what has earlier been seen at GSI, has been observed at CRYRING. The transition occurs at particle distances between 1 and $35 \mathrm{~cm}$ depending on the cooling rate, and it has been interpreted as an ordering of the ion beam. We have studied how the particle number and the momentum spread at the transition depend on the electron density in the cooler. We have also observed that the drop in momentum spread is accompanied by a reduction in Schottky power when the electron density is high.
\end{abstract}

\section{INTRODUCTION}

Some years ago, it was discovered at the ESR ring at GSI, Darmstadt how the momentum spread of beams of electron-cooled highly charged ions dropped abruptly to very small values as the beam current decayed below a certain threshold [1]. The drop in momentum spread has been seen for fully stripped ions in charge states between 18 and 92 at the ESR and later also at the SIS ring, but not at any other laboratory. The observations were interpreted as an ordering of the beams, such that the ions lined up after one another, without being able to pass each other due to the strong repulsion between the highly charged ions and small energy spread due to the cooling [2].

Similar observations have now been made at CRYRING using ${ }^{58} \mathrm{Ni}^{17+}$ ions at $8.1 \mathrm{MeV} / \mathrm{u}$ and ${ }^{129} \mathrm{Xe}^{36+}$ at 7.4 and $3.1 \mathrm{MeV} / \mathrm{u}$. These ions have sufficiently long lifetimes in the ring in order to allow reasonable observation times: with no electron current in the cooler the lifetimes were approximately $100 \mathrm{~s}$ for the $\mathrm{Ni}$ ions and $190 \mathrm{~s}$ for the 7.4-MeV/u Xe ions, mainly determined by electron capture from collisions with rest-gas molecules. Turning on the electron cooler causes ions and electrons to recombine and reduces the lifetimes. For example, the shortest lifetime of the 7.4-MeV/u Xe $\mathrm{X}^{36+}$ ions, obtained with an electron density of $2.9 \times 10^{13} \mathrm{~m}^{-3}$, was $29 \mathrm{~s}$. Because of more available beamtime, the highest-quality data were obtained with the $7.4-\mathrm{MeV} / \mathrm{u} \mathrm{Xe}^{36+}$ ions, and the following discussion will concern these ions.

\section{OBSERVATIONS}

\subsection{Particle Number at Transition}

The momentum spread of the beam was determined from the Schottky signal, and fig. 1 shows a sequence of Schottky spectra for a case where the cooling was relatively strong - the electron density was $7.3 \times 10^{12} \mathrm{~m}^{-3}$. Each spectrum, centred in the analysis to compensate for a slow frequency drift, constitutes an average of 224 individual spectra recorded by a spectrum analyzer. The horizontal scale shows the frequency relative to the $10^{\text {th }}$ harmonic of the revolution frequency.

At $t=0$, around 50,000 particles were injected, and the beam was cooled with the electron cooler. It is seen how the frequency spread suddenly shrinks approximately $125 \mathrm{~s}$ after the injection. A prominent feature of the spectra before this transition is their double-peak character, which will not be further discussed in this report. Also, it looks as if the narrow peaks have side bands at frequencies equal to those of the last double peak. These side bands are at a $50 \mathrm{~Hz}$ separation from the main peak at all harmonics, and are thus not coming from the beam, but most probably from the Schottky amplifiers.

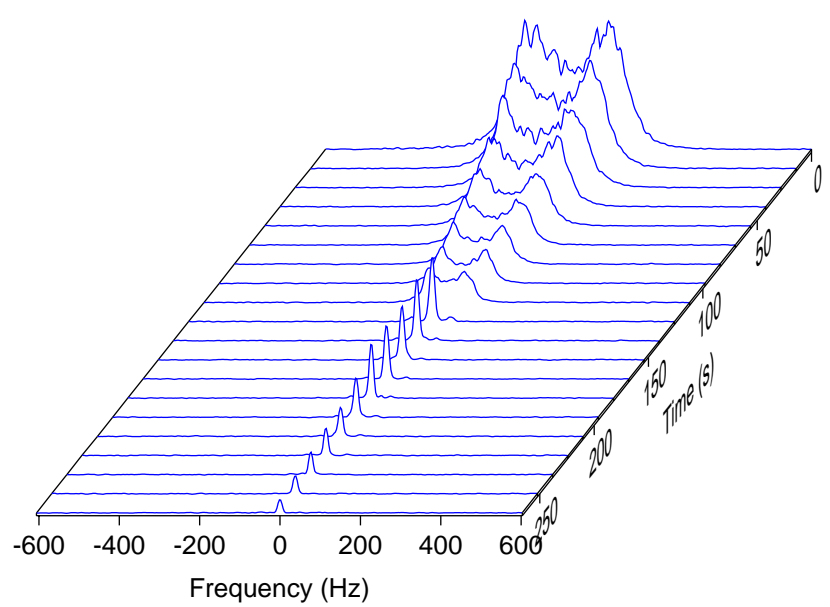

Figure 1: Sequence of Schottky spectra from beams of 7.4 MeV/u ${ }^{129} \mathrm{Xe}^{36+}$ ions.

Sequences like the one in fig. 1 were recorded for a number of different electron densities up to $2.9 \times 10^{13} \mathrm{~m}^{-3}$. Transitions to small momentum spreads were observed for electron densities down to $2.2 \times 10^{11} \mathrm{~m}^{-3}$, corresponding to an electron current of $1.7 \mathrm{~mA}$.

Fig. 2 shows the particle number at the transition to the small momentum spread as a function of the electron density. This particle number is too small to measure directly, but is calculated from the injected current, the beam lifetime (which must be known accurately since the transition occurs several lifetimes from the injection) and the time from injection to transition. At each injection, the beam current just after the acceleration, while the beam was still bunched, was determined from the intensity of a signal from an electrostatic pickup. This signal was calibrated to a DC current transformer in a separate meas- 
urement. The lifetime was measured through the count rate in a beam-profile monitor that counts ionized restgas molecules created by the ion beam. The uncertainty in the calibration of the absolute current of $\pm 10 \%$ dominates the uncertainty in the absolute particle number. Relative particle numbers are more accurate, since both the pickup signal, the profile-monitor count rate and the time to the transition could be measured with higher precision.

In fig. 2, a straight line is fitted to the data points for electron densities between $2 \times 10^{12} \mathrm{~m}^{-3}$ and $8 \times 10^{12} \mathrm{~m}^{-3}$. For our cooled beams, electron cooling and intra-beam scattering are in equilibrium. The intra-beam-scattering growth rates, horizontally, vertically and longitudinally, are then equal to the corresponding cooling rates. For simplicity, we assume that the cooling rates are proportional to the electron density and that they are independent of the emittance for the small beam emittances we are concerned with here. Furthermore, standard intra-beam-scattering theory (which, admittedly, may not be applicable to our weak beams) tells that the growth rates in each plane obey

$$
\lambda_{\mathrm{ibs}, K}=N f_{\kappa}\left(\varepsilon_{x}, \varepsilon_{z}, \varepsilon_{\mathrm{p}}\right)
$$

where $\kappa$ is $\mathrm{x}, \mathrm{z}$ or $\mathrm{p}, N$ is the number of particles and $f_{\kappa}$ are functions of the emittances. In addition, $f_{K}$ depend on the lattice parameters, the ion species and the beam energy, but these are the same for all data points in fig. 2. Thus, if standard intra-beam-scattering theory holds, a strict proportionality between electron density and particle number at the transition implies that the functions $f_{K}$ have fixed values at the transition.

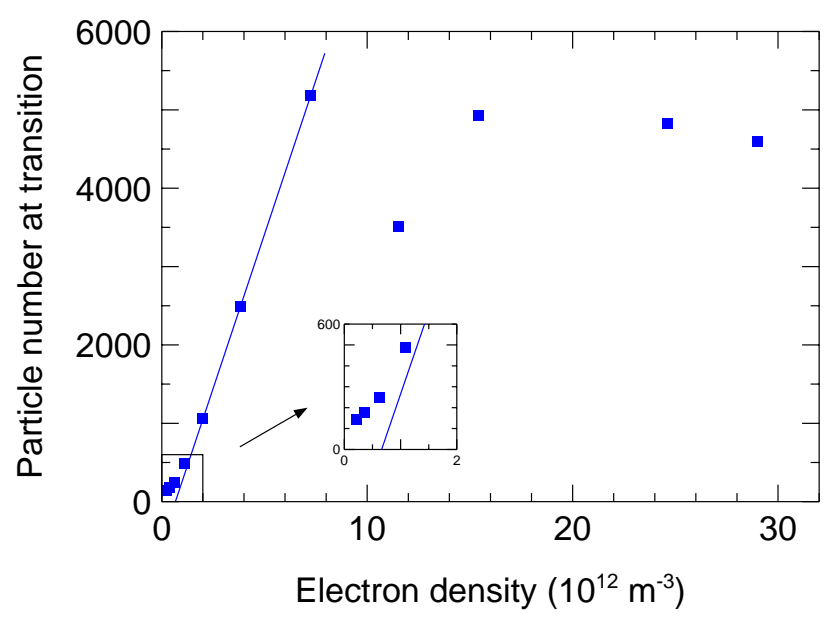

Figure 2: Number of particles in the beam at the transition to low momentum spread as a function of electron density in the cooler.

The data points in fig. 2 for electron densities above $1 \times 10^{13} \mathrm{~m}^{-3}$ have relatively constant particle numbers, which seems to indicate that ordered states do not form at particle numbers above approximately 5000. Although one could argue that the cooling conditions may have been less favourable at higher densities, the electronbeam quality and alignment could to some extent be checked by monitoring the ion-electron recombination rate through the beam lifetime. Except for the fact that the three highest points were taken with an electron-beam expansion of a factor 25 instead of 100 as used otherwise, reducing the recombination rate to approximately one half, the recombination rate was essentially proportional to the electron density. More studies are needed before a definite conclusion can be drawn about this apparent limit.

As shown in the inset in fig. 2, ordering appeared at electron densities down to $2.2 \times 10^{11} \mathrm{~m}^{-3}$ and 145 particles in the ring. This means that intra-beam scattering in some sense "works" even when the distance between the particles in the $52-\mathrm{m}$-circumference ring is as large as $35 \mathrm{~cm}$, although the deviation from the straight line at very low electron densities might be interpreted as the intra-beam scattering getting weaker. Although the particle number at transition levels off at about 125 particles as the cooling becomes very weak, no transition could be observed at the lowest electron density used, $6 \times 10^{10} \mathrm{~m}^{-3}$.

\subsection{Momentum Spread at Transition}

From Schottky sequences like the one in fig. 1, the momentum spread of the beams can be extracted as functions of particle number and electron density. This is shown in fig. 3 for a range of electron densities between $2.2 \times 10^{11} \mathrm{~m}^{-3}$ and $7.3 \times 10^{12} \mathrm{~m}^{-3}$ (the same densities as in fig. 1). Although the individual data points scatter somewhat, the particle number at the transition can be determined quite accurately, as discussed above. The momentum spread at the transition (where slanted and vertical lines meet) is thus also quite accurate and another straight line is drawn through these points.

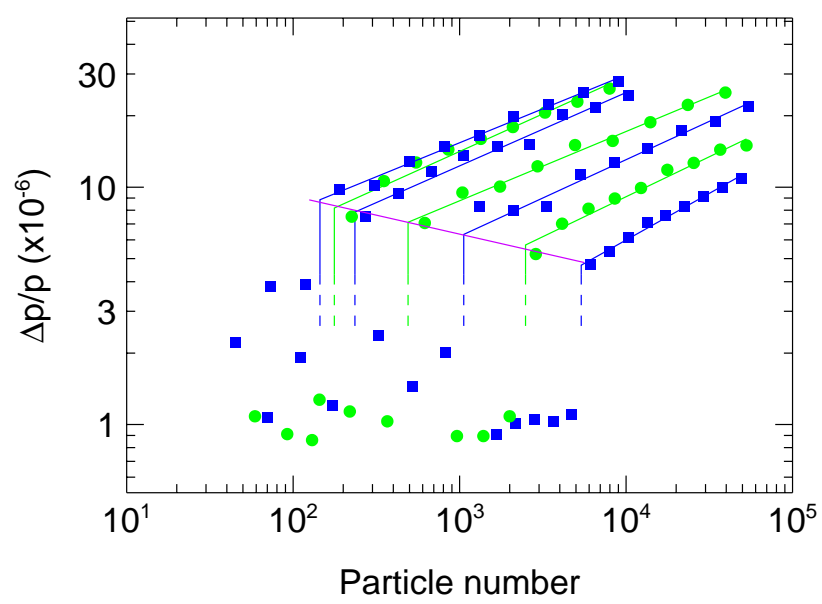

Figure 3: Momentum spread for different electron densities as function of particle number. Different symbols are used just to help identifying the points that belong to the same electron density.

From the average slope of the fitted lines it is found that the momentum spread scales with particle number as

$$
\Delta p / p \sim N^{0.32+0.03} .
$$


This can be compared to the power of 0.3 stated in ref. [1].

The width of the Schottky peaks after the transition indicates a relative momentum spread of around $1 \times 10^{-6}$ (the higher values at low particle numbers in fig. 3 are most probably due to noisier signals). This is only an upper limit of the true momentum spread, however, since ripple on the power supplies to the dipole magnets in the ring and to the cathode of the electron cooler (which defines the electron energy) should contribute at least that amount of widening of the peaks.

\subsection{Schottky Power}

The Schottky power at the $10^{\text {th }}$ harmonic of the revolution frequency is plotted in fig. 4 for one high and one low electron density. Open symbols show the power before the transition and filled symbols represent the ordered beam. With a low electron density of $2.0 \times 10^{12} \mathrm{~m}^{-3}$ (blue circles), the Schottky power is almost exactly proportional to the particle number both before and after the transition (the line drawn by hand through the circles has unit slope). When the electron density is high, $7.3 \times 10^{12} \mathrm{~m}^{-3}$, there is a reduction in Schottky power before the transition associated with the appearance of coherent oscillations in the beam (manifested by double peaks in the spectra). Also, the transition to the ordered state is accompanied by a drop in Schottky power, which possibly can be interpreted as the appearance of additional correlation between the ions.

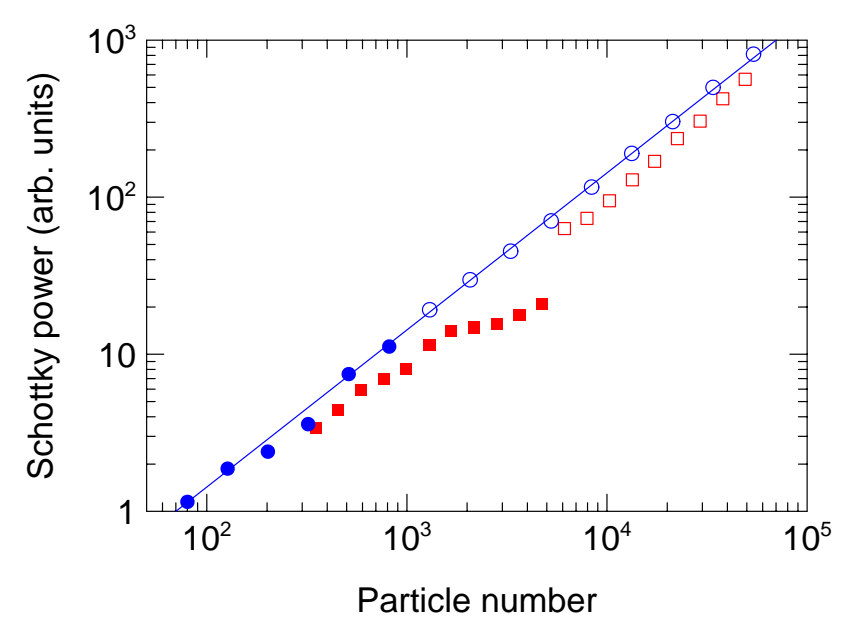

Figure 4: Schottky power as function of particle number for electron density $2.0 \times 10^{12} \mathrm{~m}^{-3}$ (blue circles) and $7.3 \times 10^{12} \mathrm{~m}^{-3}$ (red squares). The line, which has slope equal to one, is drawn by hand through the blue circles.

\section{INTERPRETATIONS}

The observations at GSI were interpreted as a transition to an ordered, liquid-like state. Liquid order can be expected to appear when the plasma parameter $\Gamma$, defined as the ratio between the mean potential energy of one particle in the field of its neighbour and the particles' mean kinetic energy $k T$, i.e.,

$$
\Gamma=Z^{2} q^{2} /\left(4 \pi \varepsilon_{0} d k T\right),
$$

where $d$ is the mean distance between particles, approaches unity.

In our case, at 5000 particles, $d$ is equal to $1 \mathrm{~cm}$ and the mean potential energy is $0.2 \mathrm{meV}$. At 145 particles, the lowest number where a transition is seen, the mean distance between the particles is already 3.5 times the diameter of the stainless-steel beam pipe, and one would expect that the particles are quite well shielded from one another. The longitudinal beam temperature (multiplied by $k$ ), as obtained from the momentum spread, ranges between approximately 50 and $150 \mathrm{meV}$ just before the transition. After the transition, an upper limit for the longitudinal beam temperature is $2 \mathrm{meV}$.

The transverse beam dimensions are below the resolution of our beam-profile monitors, even at the very lowest electron densities when cooling is weakest. With a resolution of around $0.5 \mathrm{~mm}$ FWHM, only an upper limit of $3 \mathrm{eV}$ can be given for the transverse beam temperature. However, it is reasonable to assume that, as long as intrabeam scattering is effective, transverse and longitudinal temperatures are of similar magnitude. After the transition, when intra-beam scattering is strongly suppressed, the transverse beam temperature should reflect the effective electron temperature, which is in the range around $1 \mathrm{meV}$. Thus one can conclude that the plasma parameter is much smaller than 1 before the transition, but that it very well could be close to 1 in the ordered state.

As confirmed by simulations [2], the characteristic feature of the beams in ESR seems to be that the particles are arranged in a linear configuration, where the strong repulsion between the highly charged ions and their low relative energy prevent them from passing each other. Since the observations at CRYRING agree quite well with those at the ESR in terms of particle numbers and momentum spreads, it appears clear that the same explanation holds in our case.

This explanation is also supported by detailed MonteCarlo simulations of beam ordering in CRYRING [3]. Linear chains of ions (and also more complex structures) were found to appear in these simulations, even if they were performed with higher particle numbers and higher cooling rates than those used in the present experiments.

\section{REFERENCES}

[1] M. Steck et al., Phys. Rev. Lett., 77 (1996) 3803

[2] R. W. Hasse, Phys. Rev. Lett., 83 (1999) 3430

[3] J. Beebe-Wang, N. Elander and R. Schuch, Phys. Rev E 50 (1994) 4073. 